\title{
Nature urbaine et urbanité dans la station touristique de Salou (Espagne), au travers de l'étude : d'un parc-promenade, d'un paseo, d'un parc urbain
}

Jean Rieucau

\section{OpenEdition}

Journals

Édition électronique

URL : http://journals.openedition.org/echogeo/12191

DOI : 10.4000/echogeo.12191

ISSN : 1963-1197

Éditeur

Pôle de recherche pour l'organisation et la diffusion de l'information géographique (CNRS UMR 8586)

Référence électronique

Jean Rieucau, « Nature urbaine et urbanité dans la station touristique de Salou (Espagne), au travers de l'étude : d'un parc-promenade, d'un paseo, d'un parc urbain », EchoGéo [En ligne], Sur le Vif, mis en ligne le 30 novembre 2010, consulté le 30 avril 2019. URL : http://journals.openedition.org/ echogeo/12191; DOI : 10.4000/echogeo.12191

Ce document a été généré automatiquement le 30 avril 2019.

\section{(c) (1) ()}

EchoGéo est mis à disposition selon les termes de la licence Creative Commons Attribution - Pas d'Utilisation Commerciale - Pas de Modification 4.0 International 


\title{
Nature urbaine et urbanité dans la station touristique de Salou (Espagne), au travers de l'étude : d'un parc-promenade, d'un paseo, d'un parc urbain
}

\author{
Jean Rieucau
}

\section{Introduction}

1 Au début du XXI e siècle, les stations touristiques littorales sont marquées par de nouvelles pratiques récréatives et résidentielles. Celles-ci entrainent une convergence touristicorésidentielle, caractérisée par la mise en place de continuums entre espaces de vie et espaces récréatifs. Sur les littoraux de l'Arc méditerranéen, nombre de stations stricto sensu se muent en villes-stations, mêlant touristes et résidents permanents, aux attentes, aux rapports différents avec l'espace public, en particulier avec la nature urbaine.

2 L'environnement de la ville déploie une forte diversité, faite de l'air, de l'eau, du minéral, $\mathrm{du}$ sol, du vivant. Les stations touristiques côtières introduisent une complexité environnementale supplémentaire : le contact de la ville avec la nature marine vivante en terminaison d'écoumène, par l'intermédiation des plages. ${ }^{1}$ La place de la nature dans la ville, portée par les concepts d'écoquartier, d'urbanisme durable, de ville "renaturée ", devient un enjeu fondamental de la gouvernance urbaine. L'urbanité d'une station touristique, qui permet l'échange, le contact, la mixité sociétale, se construit de manière croissante autour de la conception et des usages citadins de ses espaces publics naturés.

Comment, dans les stations touristiques littorales méditerranéennes espagnoles, caractérisées depuis les années 1955-1960 par un tourisme de masse de sol y playa ${ }^{2}$, jugé agressif pour l'environnement et les paysages côtiers, recréer de la nature dans la ville? Pourquoi et comment, la station de Salou (carte 1 et illustration 1), lieu touristique situé 
sur la Costa Daurada au sud de Tarragone en Catalogne, à proximité du second parc thématique européen (Port Aventura), a-t-elle systématisé davantage que ses concurrentes (Benidorm, Gandía, Cullera, Torrevieja) (Rieucau, 2006), l'aménagement de promenades littorales et intérieures, pour leur rôle naturant et socialisant?

4 Après une réflexion sur les références paysagères de la ville littorale qui mêlent urbanité, ruralité et maritimité, l'étude positionnée au carrefour entre la géographie urbaine et du tourisme, s'interrogera sur le rôle assigné à plusieurs formes urbaines naturées : création ex nihilo de paseos, maintien d'un parc urbain, aménagement d'un parc-promenade situé en front de mer.

\section{Carte 1 - Espaces publics naturés à Salou}

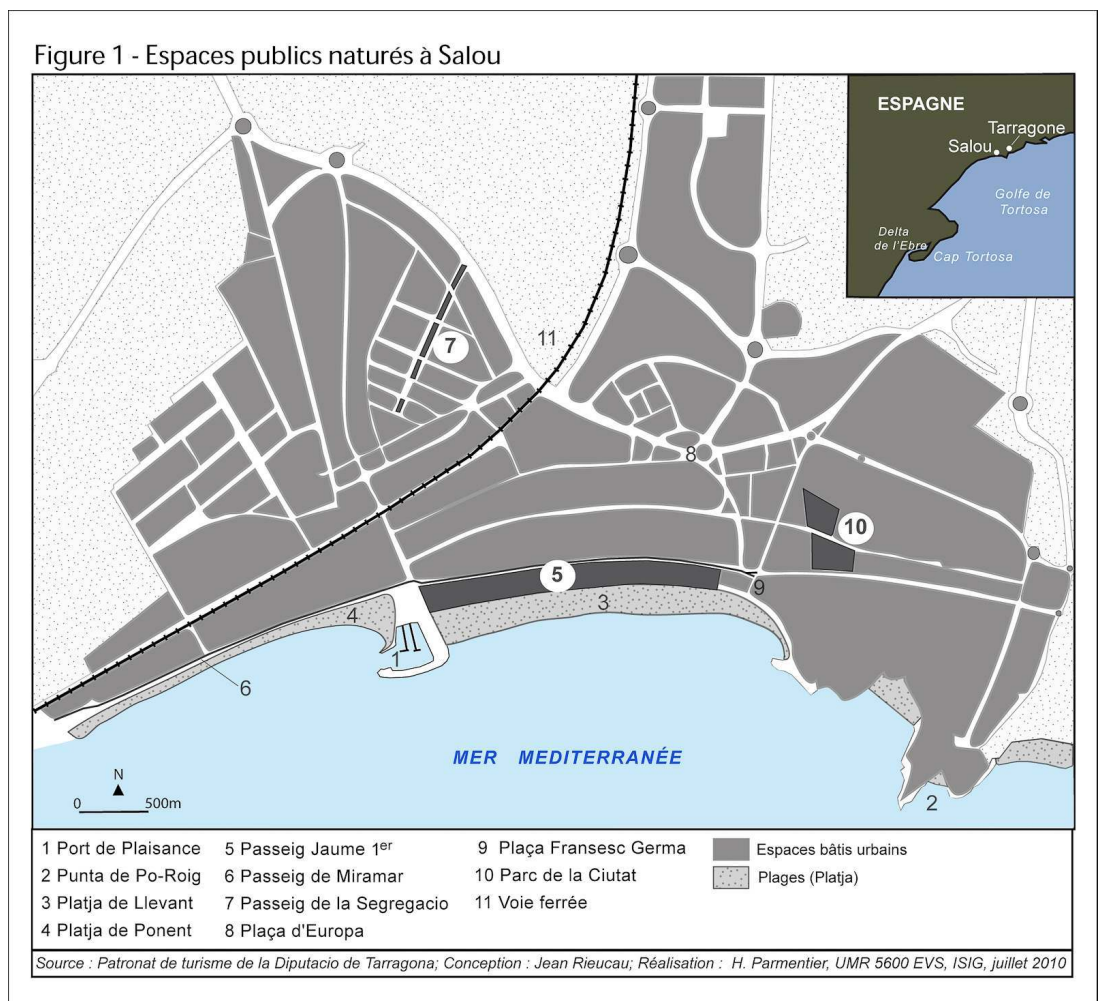




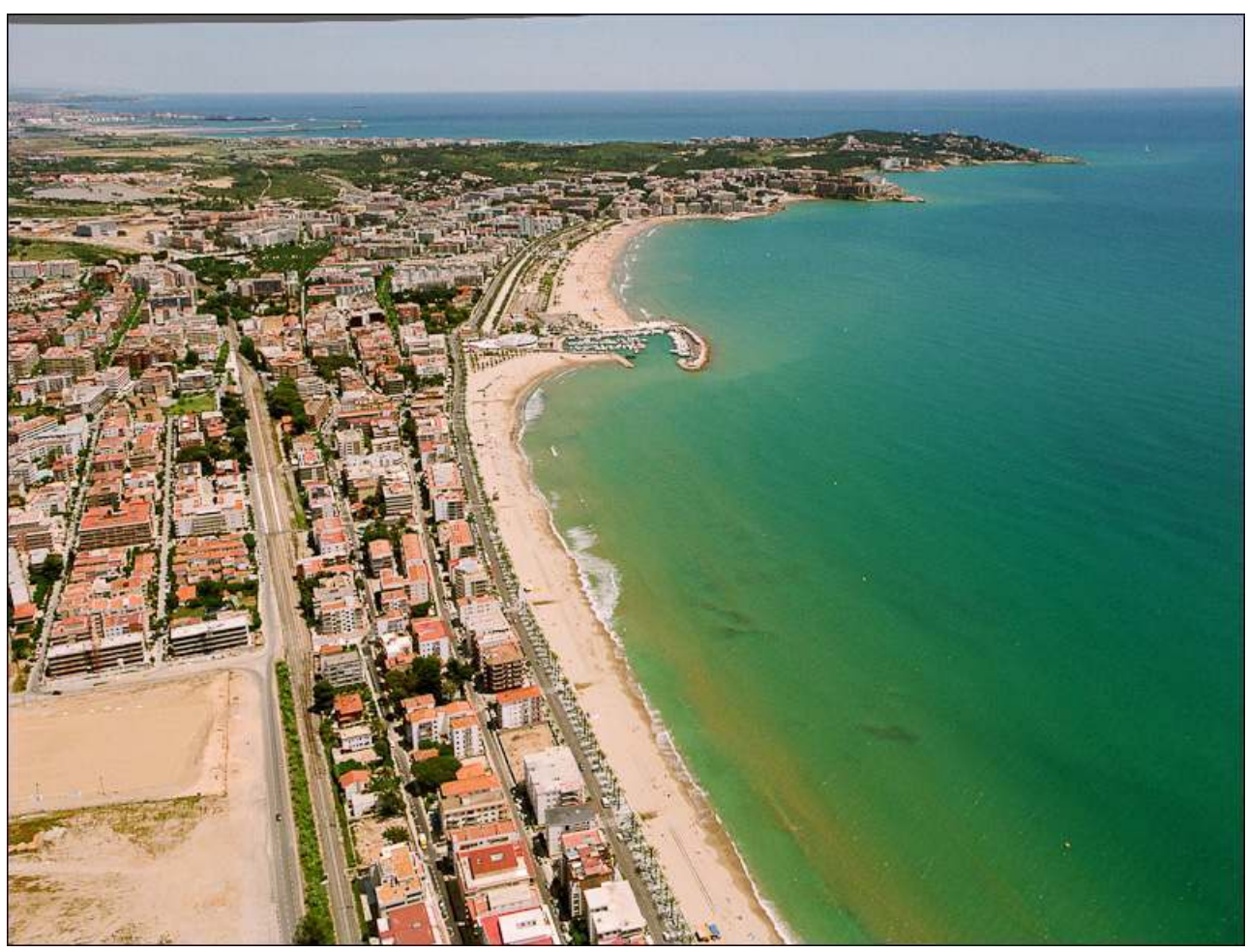

Auteur : Mairie de Salou.

\section{Lieux de nature et urbanité dans la ville}

\section{Une nature urbaine?}

6 La ville a longtemps représenté un espace de non nature, le territoire de l'antinature (Arnould, 2006), l'antipode absolu de la nature (Estebanez, 2006), le domaine du dénaturé, un lieu artificiel (Arnould, op. cit.), une réalité hybride. « Aujourd'hui, la société occidentale trouve un intérêt pour certaines formes de vie sauvage dans les parcs publics» (Dorier Apprill, 2006). Il se caractérise par un goût pour une " nature spontanée » qui se traduit par la valorisation de la flore indigène (Dorier Apprill, op. cit.).

7 Urbanité et espaces publics

L'urbanité, assimilée par dérive à une ambiance urbaine raffinée qui faciliterait la familiarisation avec «l'usage du monde» (Merlin, Choay, 1996) prend place dans des espaces publics, permettant convergence, interaction, coprésence entre des individus. Ces lieux de sociabilité ouverte facilitent la rencontre éphémère, dans l'anonymat, des personnes issues de groupes sociaux, de professions, d'ethnies, de confessions différentes (Ghorra Gobin, 2001). L'espace public suppose que soient réunis trois principes élémentaires : une accessibilité maximale, un fort potentiel de rencontre de l'autre, une mise en scène de l'altérité (Berdoulay, Morales, 1999). Le rapport de la ville à la nature participe d'une nouvelle urbanité ou "nature d'urbanité » (Bonnin, 2010), créant des synergies entre le bâti et le végétal, rapprochant le logement et les espaces verts urbains (jardin, parc, promenade).

8 Le jardin public, siège de la nature urbaine et lieu historique d'urbanité 
9 Le jardin, censé cristalliser l'urbanité (Lévy, Lussault, 2003), évolue du domaine privé au domaine public, à partir du siècle des Lumières et se caractérise comme l'espace d'une urbanité spécifique, plus intime que celle des rues et des places (Lévy, Lussault, op. cit.). L'art et la culture des jardins, en Europe, ont longtemps représenté dans la ville, les principaux lieux de biodiversité contrôlée et dirigée (Arnould, op.cit.). Au sein de la nature urbaine, le jardin public (d'ornement, botanique, exotique, paysager) occupe une place centrale (Gillot,2006). Le jardin qui alimente un puissant imaginaire (naturaliste, hygiéniste, environnementaliste), représente souvent dans l'inconscient collectif citadin, la nature dans la ville (Lévy, Lussault, op. cit.).

Au XIX ${ }^{e}$ siècle, le parc public ${ }^{3}$, dans la continuité historique de Central Park à New York, de Hyde Park à Londres, du parc Montsouris à Paris, a permis de préserver et de réintroduire de la nature dans les villes (Arnould, op.cit.), de contribuer à leur embellissement, d'en améliorer l'hygiène. Au XXe siècle, cet espace vert, à la frontière de la nature et de la société (Gillot, op.cit.), entre nature et culture, fonde largement la pensée paysagiste (Donadieu, 2005).

$11 \mathrm{Au}$ début du XXe siècle,les jardins publics revêtent encore des vertus sociales, morales, esthétiques (Gillot, op. cit.). Selon certains auteurs, le jardin témoigne à sa manière, du processus de civilisation des moeurs (Lévy, Lussault, op. cit.), sorte de refuge à l'abri des violences, des incivilités, face aux dangers de la ville (Merlin, Choay, op. cit.). Les espaces verts facilitent les situations d'isolement, de retranchement et constituent des lieux de rencontres (Merlin, Choay, op. cit.).

$12 \mathrm{Au}$ début du XXI ${ }^{\mathrm{e}}$ siècle, l'existence d'espaces publics (places, parcs, jardins, édifices publics), au même titre que le degré de mixité sociale résidentielle, peuvent constituer des indicateurs de l'urbanité des villes (Dorier-Apprill, op. cit.).

Les références paysagères contemporaines de la station littorale : entre urbanité, ruralité et maritimité

14 Il existe, dans la société occidentale, une demande sociale de paysage, de pratique de nature (Donadieu,op.cit.), que les paysagistes qualifient de besoin de vert, de désir de paysage, de campagne. Une demande sociale en lieux de nature, ainsi qu'un marché du paysage (Donadieu, op. cit.), parcourent l'ensemble des villes. Les travaux des paysagistes nous permettent de comprendre que deux types de motifs, de références paysagères, interfèrent dans les villes (Donadieu, op. cit.), ceux relevant de la ruralité, ceux participant de l'urbanité, auxquels s'ajoutent, pour les villes côtières, ceux qui mettent en scène la maritimité (Péron, Rieucau, 1996).

\section{Les motifs de l'urbanité}

Les motifs de l'urbanité participent de l'art urbain (Merlin, Choay, op. cit.) représenté par la rue, la place, le jardin public, complété dans le bassin méditerranéen par les esplanades, les allées, les promenades (Rieucau, op. cit.). Au sein de ces formes urbaines, jardins publics et espaces verts occupent, dans l'histoire des villes, une place centrale pour leur caractère à la fois naturant et socialisant.

\section{Les motifs de la ruralité dans l'espace urbain}

Les motifs de la ruralité à l'intérieur de la ville résident dans l'usage de l'arbre urbain (illustration 2), de la haie au sein des jardins, de la prairie au sein des parcs (Donadieu, op. cit.) et des espaces forestiers ${ }^{4}$. A Salou, en Catalogne, ville-station balnéaire à double fréquentation nationale et internationale, les promenades des extensions urbaines 
récentes, seulement fréquentées par des populations catalanes résidentes, mettent en avant la ruralité par le recours à une végétation indigène (vieux oliviers dits « monumentaux », cyprès, acacias, eucalyptus, lauriers roses) (illustration 3). Ces espaces verts, qui ne contribuent pas à la construction de l'image touristique, excluent l'usage des palmiers et de végétaux exotiques, à la différence du vaste parc Jaume Ir (illustration 4) aménagé en bord de mer, vitrine touristique de la station et principal lieu de déambulation des villégiateurs.

Illustration 2 - Mise en place d'une trame verte, au moyen de la plantation d'oliviers, et d'eucalyptus, encadrant le paseo de la Segregació

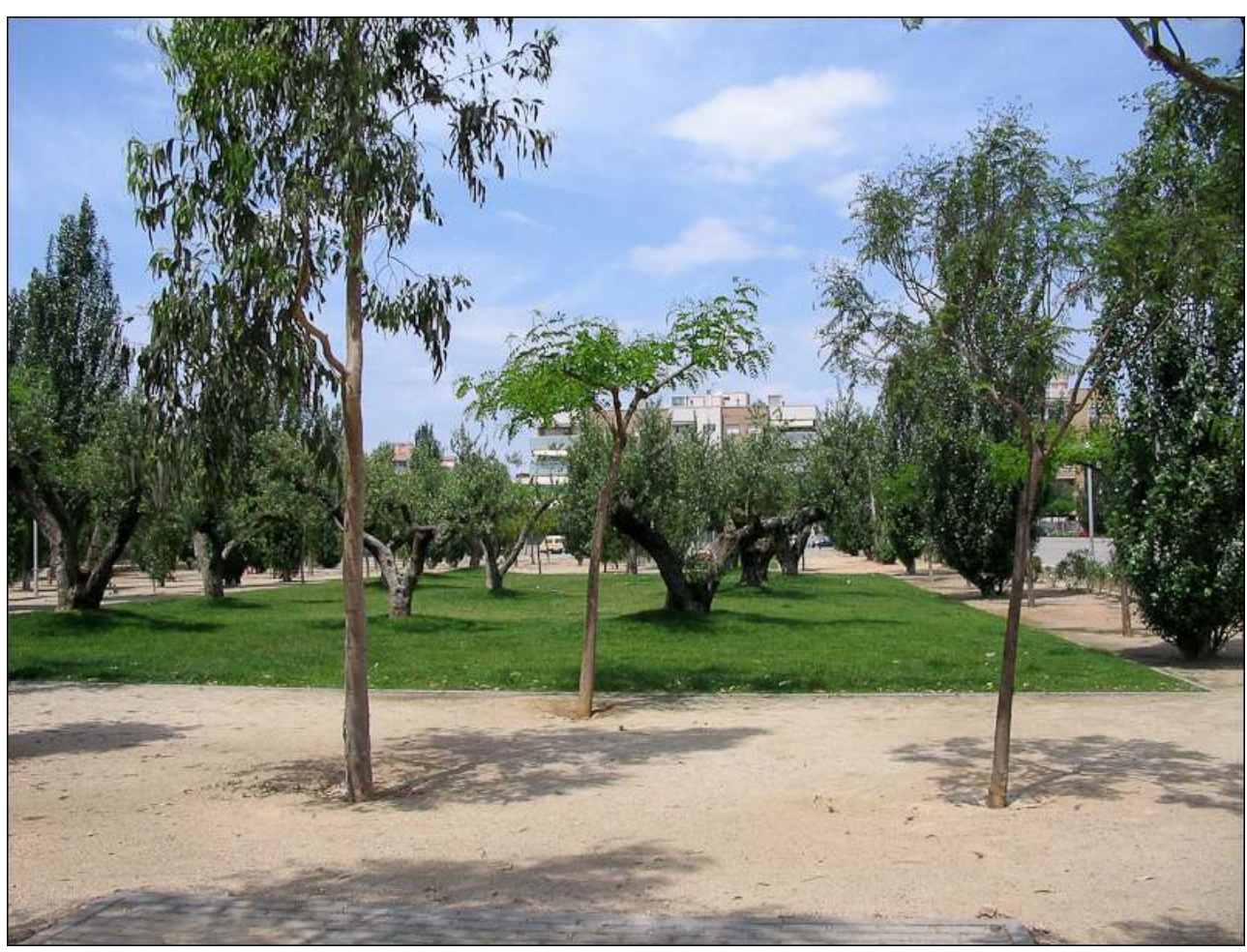

Auteur : Jean Rieucau, mai 2006. 
Illustration 3 - Olivier « monumental » sur le paseo de la Segregació

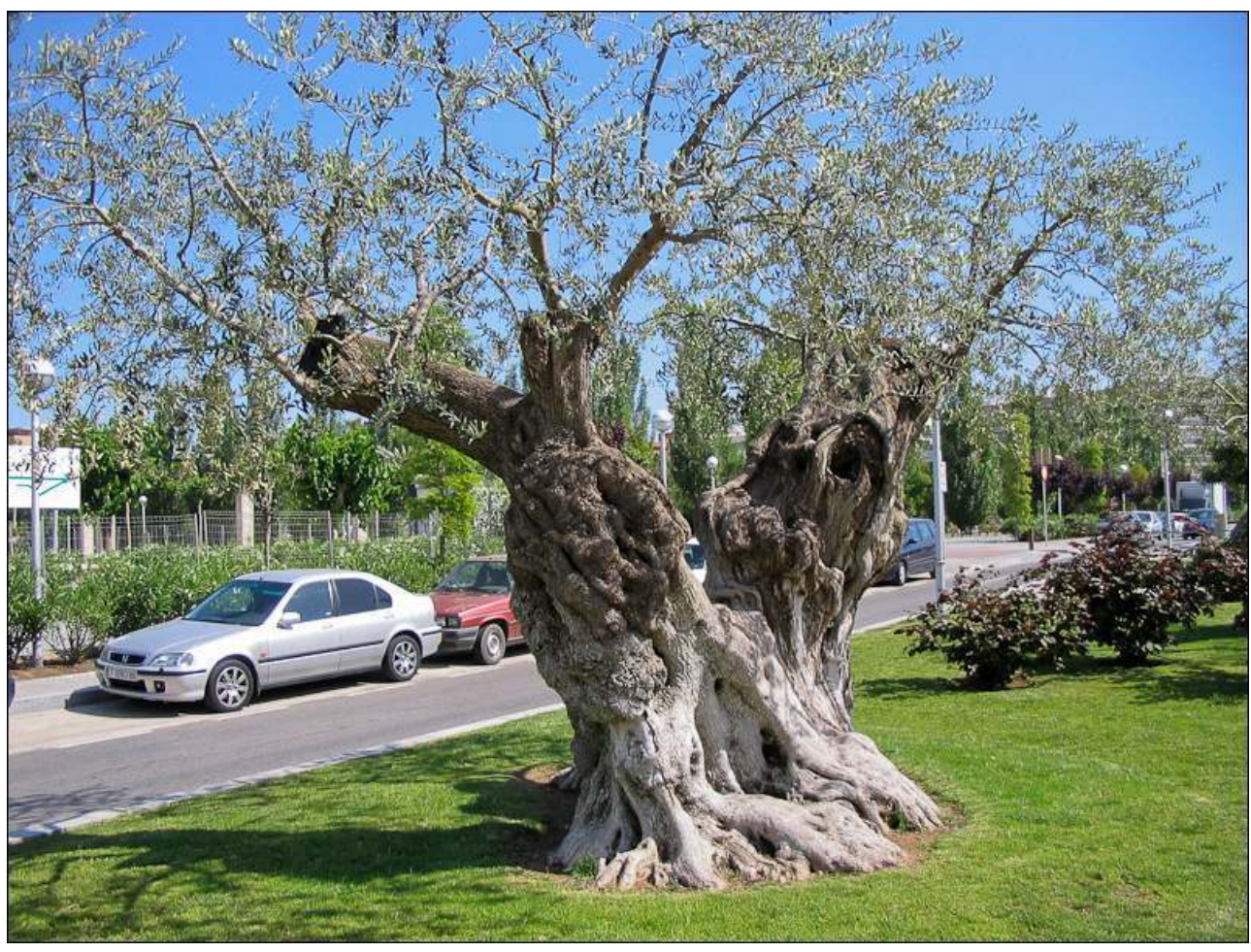

Auteur : Jean Rieucau, mai 2006.

Illustration 4 - Le parc-promenade Jaume ler

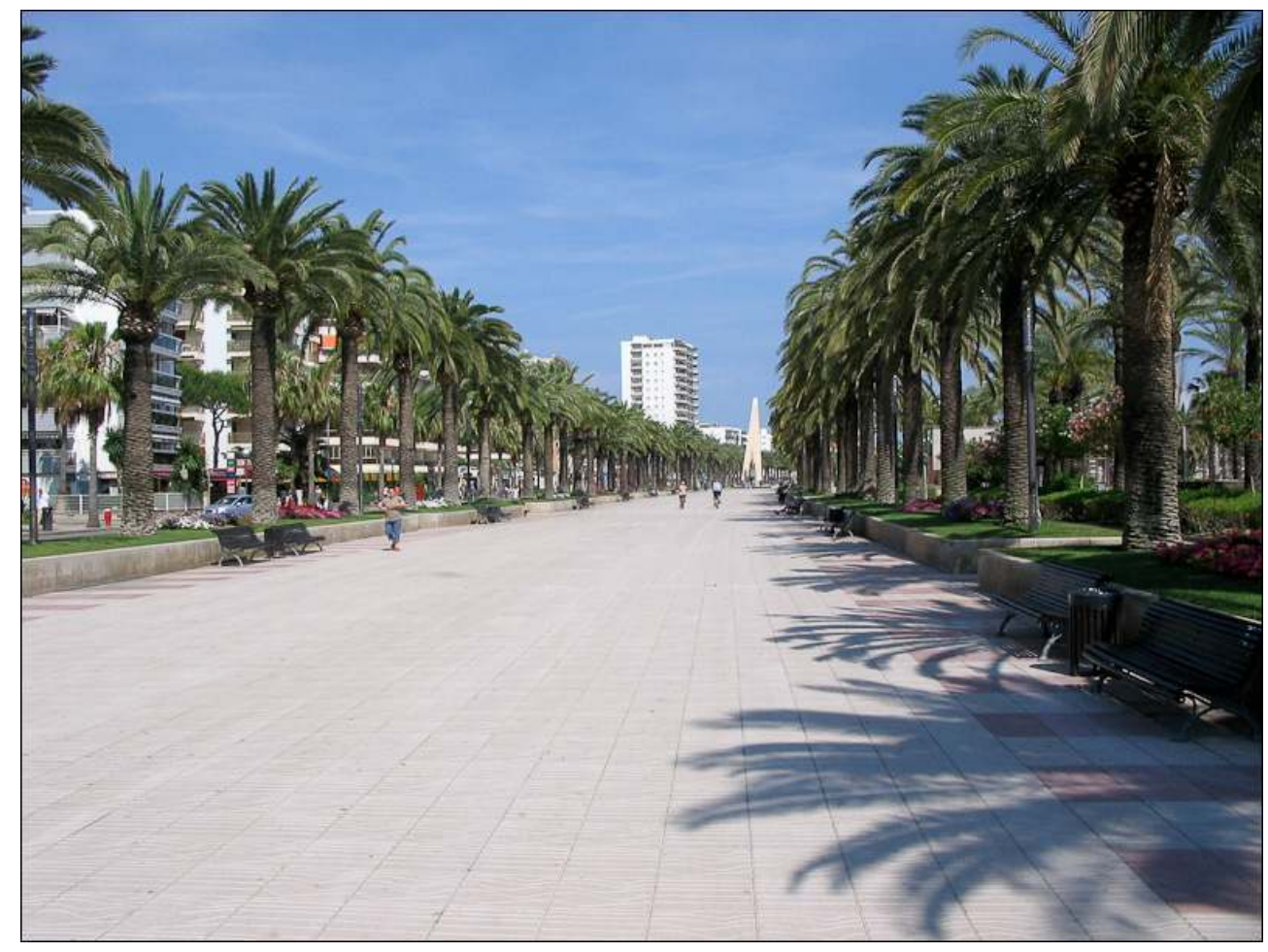

Auteur: Jean Rieucau, mai 2006. 


\section{Les motifs de la maritimité dans la station touristique littorale}

Villes et stations touristiques littorales complexifient le classique binôme paysager ruralité/urbanité, en introduisant la variable maritime. Les motifs de la maritimité (Péron, Rieucau, op.cit.), se fondent sur la promenade maritime, la plage, le port de plaisance ${ }^{5}$, la mise en scène des jetées, des digues et des môles, la patrimonialisation des barques (illustration 5) et des engins de pêche (illustration 6). Dans l'histoire urbaine, les allées, les cours, dans les villes continentales, ouvrent la ville vers la campagne (Dorier Apprill, op. cit.). De la même manière, la promenade littorale permet de voir la mer, d'observer le paysage et le panorama marin tout en déambulant (Rieucau, op.cit.). L'existence d'un véritable port de pêche au sein d'une ville touristique peut rehausser sa

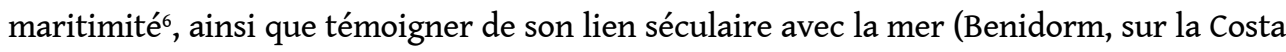
Blanca). Enfin, dans les villes maritimes et au sein des stations littorales, la plage occupe une place à part tel un espace atypique (Lageiste, Rieucau, 2008). Comme espace récréatif et d'interrelations sociétales compenserait-il, remplacerait-il, l'absence voire la moindre importance en superficie, en variété, des jardins et des parcs publics ? (Coralli, 2007).

Illustration 5- Mise en avant de la fonction halieutique passée, par la patrimonialisation des barques de pêche et par la reconstitution de jetées

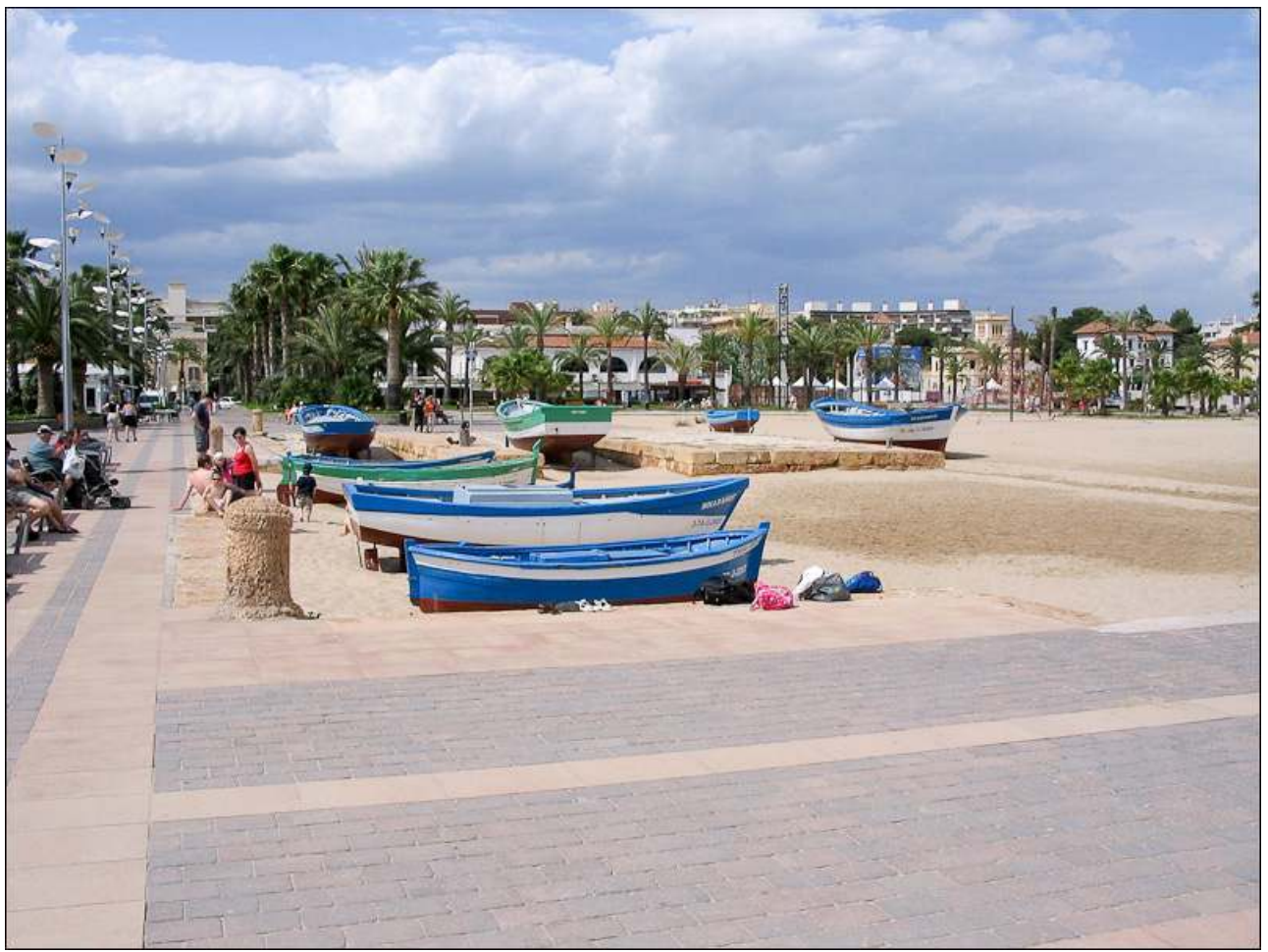

Auteur : Jean Rieucau, mai 2006. 


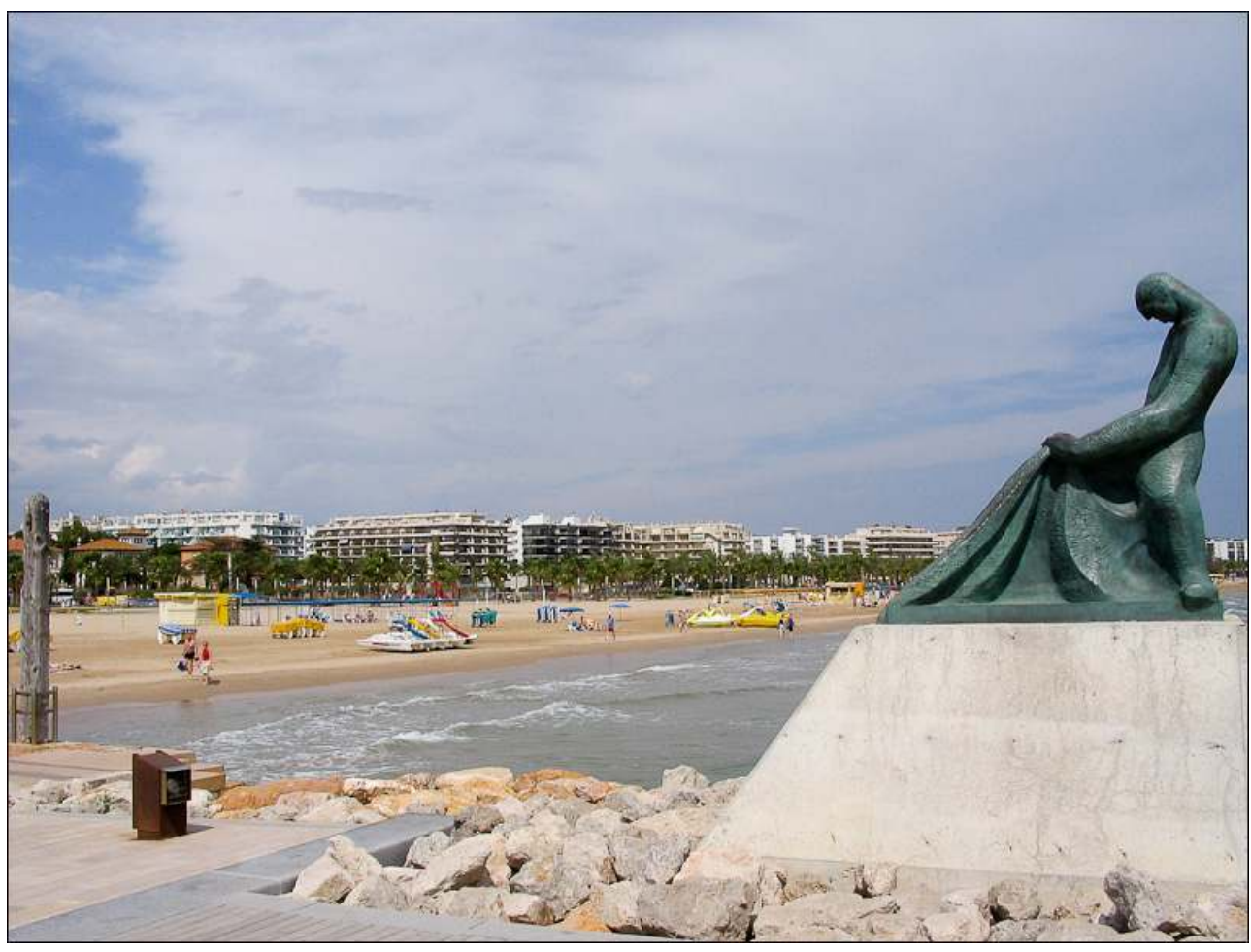

Auteur : Jean Rieucau, mai 2006.

\section{Lieux de nature publics et urbanité à Salou}

\section{Renouvellement urbain et renaturation}

La ville de Salou s'est lancée dans une opération de renouvellement urbain, pour rétablir les équilibres internes de la ville entre les espaces bâtis et les espaces verts. Le renouvellement urbain procède d'une reconstruction de la ville sur elle même, au moyen de la reconquête, la récupération de terrains en friche, de la restructuration des quartiers par la réhabilitation ou par des opérations de démolition. La municipalité a conduit une active politique de récupération d'espaces urbains aménagés hâtivement pour le tourisme de masse dans les années 1960, pour mettre en place des espaces publics de nature, au moyen d'espèces locales dites végétation contextuelle (oliviers, lauriers, amandiers...).

Les paysagistes, pour contenir l'urbanisation littorale, atténuer l'impression de massification touristique, dans l'ensemble des stations balnéaires, procèdent à une récupération du domaine public littoral. La récupération urbaine a porté sur les hauts de plage, le front de mer et sur les terrains de camping enchâssés dans le tissu urbain. Le long du linéaire côtier (illustration 7), les pouvoirs publics favorisent la mise en place de zones vertes côtières par la destruction et l'expropriation des constructions illégales. Cette politique s'accompagne également de la récupération des espaces non bâtis (pinèdes côtières, vallons couverts de garrigue, protection d'espèces locales de palmiers, de petite taille, résistantes aux embruns). Les plans d'extension de la ville au nord reposent sur la mise en place d'une trame verte fondée sur des espèces autochtones (oliviers, arbres 
fruitiers, jeunes pins, peupliers), servant de transition, d'annonce végétale avec les espaces ruraux périphériques.

Illustration 7 - Mise en place d'une banquette piétonne (Ronda de mar) sur la côte rocheuse

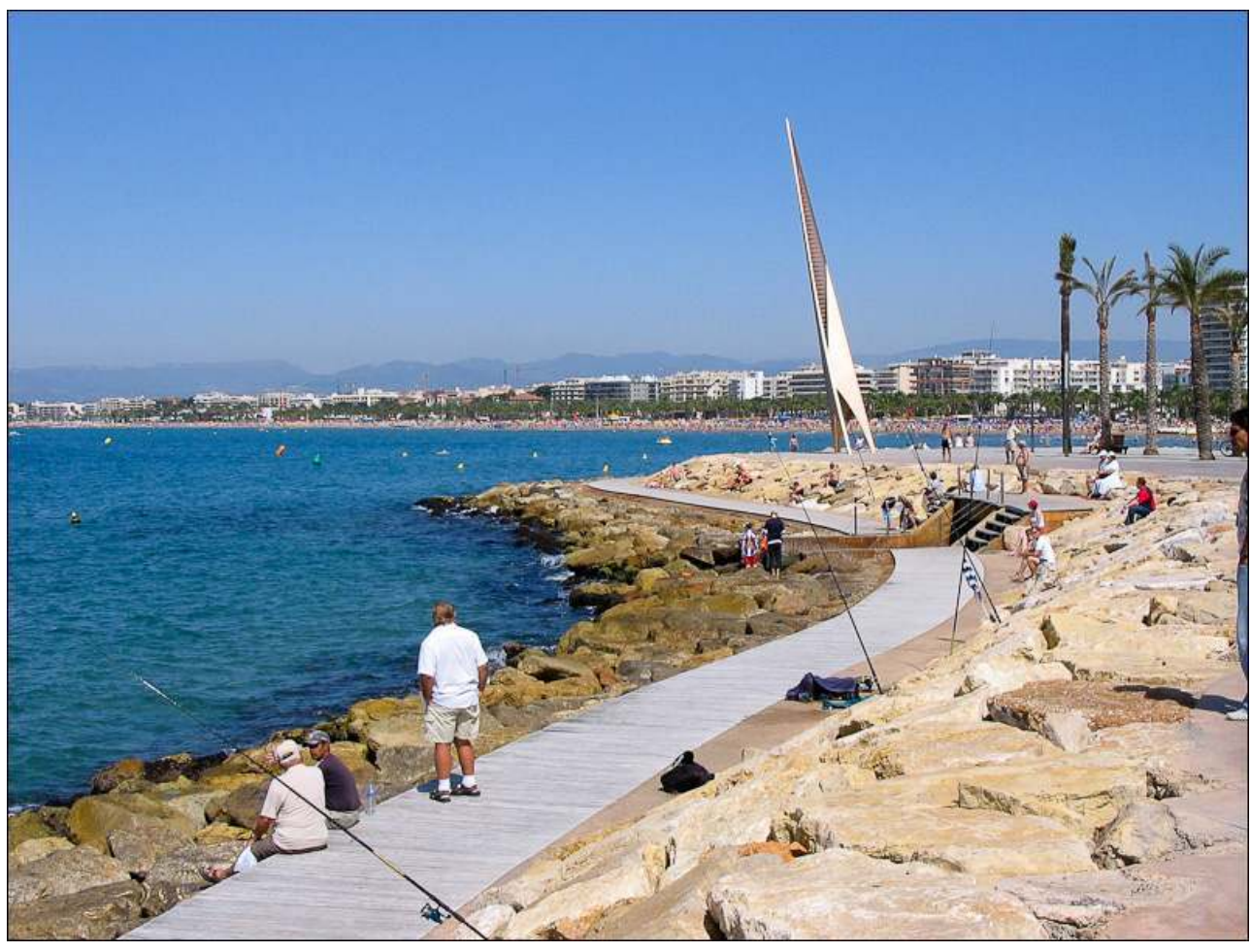

Au deuxième plan, vue sur le parc-promenade Jaume $\mathrm{I}^{\mathrm{er}}$

Auteur : Jean Rieucau, mai 2006.

\section{Le parc-promenade Jaume $\mathrm{I}^{\mathrm{er}}$ : renouvellement urbain et renaturation du front de mer touristique}

A Salou, le parc-promenade Jaume $\mathrm{I}^{\mathrm{er}}$, aménagé en front de mer, situé au centre de la station (carte 1), se déploie sur un kilomètre de long et 120 mètres de large, encadré par deux parcs de stationnement. Dans les années 1970, l'actuel parc est occupé à la fois par un vaste parc de stationnement et des zones foraines. Au début du XXI ${ }^{e}$ siècle, cet espace de déambulation résulte d'une politique de récupération puis de renaturation urbaine d'espaces touristiques aménagés anarchiquement entre 1955 et 1960. Cette renaturation urbaine s'accompagne également sur la grève attenante d'une végétalisation du haut de plage (Platja Ponent) (carte 1), (illustration 8), aux dépens d'hôtels construits dans les années 1955.

Le parc-promenade ${ }^{7}$ se compose de cinq lignes de palmiers parallèles (espèce végétale introduite entre 1880 et 1920), de différentes tailles, créant une ambiance de boisement diffus, peu ombragé, aux espèces faiblement variées, majoritairement des lauriers roses et des fleurs. La diversité végétale est faible, dépourvue d'espèces locales, à la différence des zones résidentielles (figuiers, oliviers, cactées). Le rapport entre le minéral et le végétal ${ }^{8}$ est à l'avantage du premier (illustration 9) en raison de la place occupée par le mobilier urbain, les lampadaires métalliques, par les locaux d'entretien en bois (illustration 10) et par le pavement minéral clair qui réfléchit la lumière. 
Illustration 8 - Contact entre le parc-promenade et la plage : débordement des surfaces engazonnées sur le haut de plage

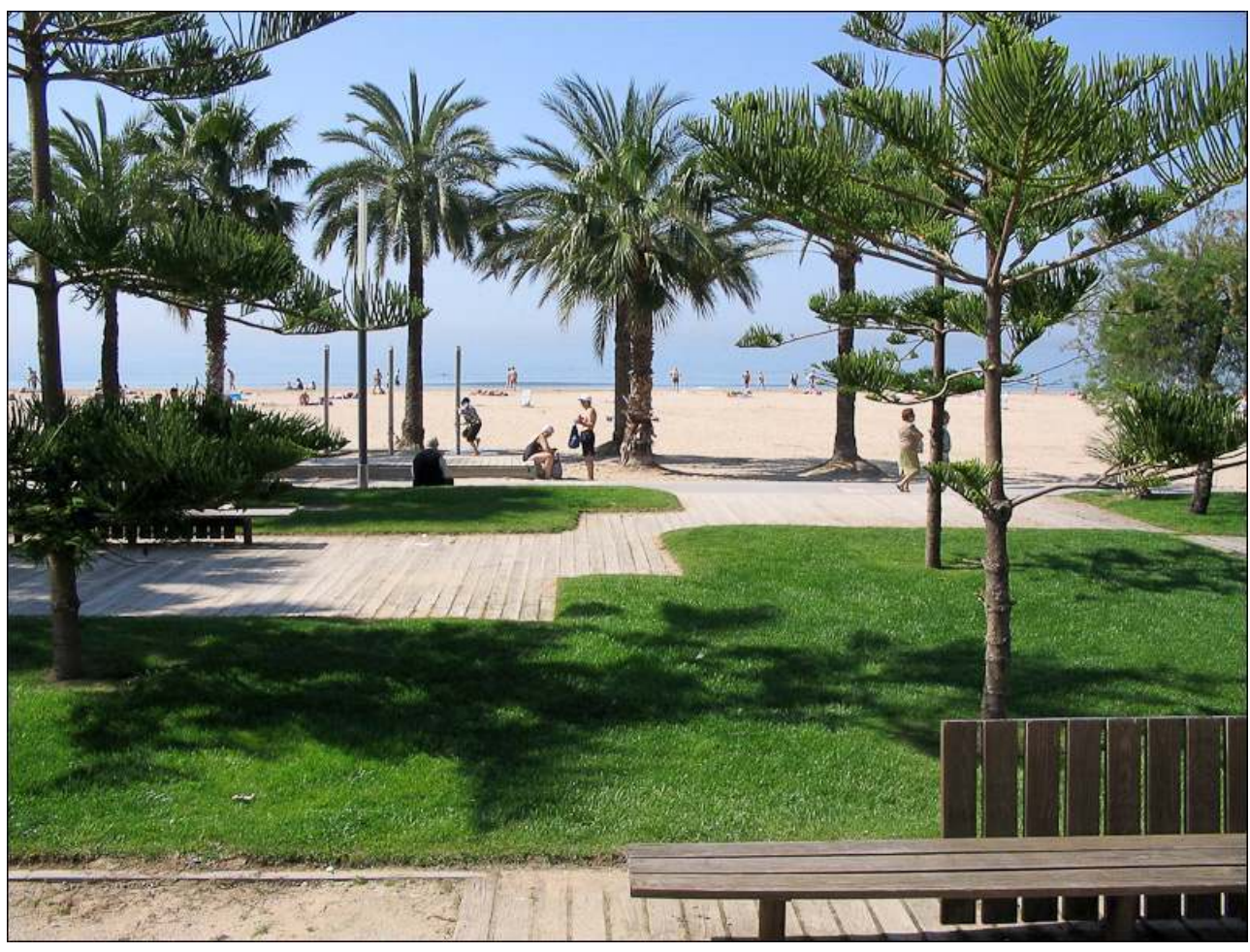

Auteur : Jean Rieucau, mai 2006

Illustration 9 - La promenade centrale du parc Jaume ${ }^{\text {er }}$ : minéralité, monumentalité de la conception, faible végétalisation

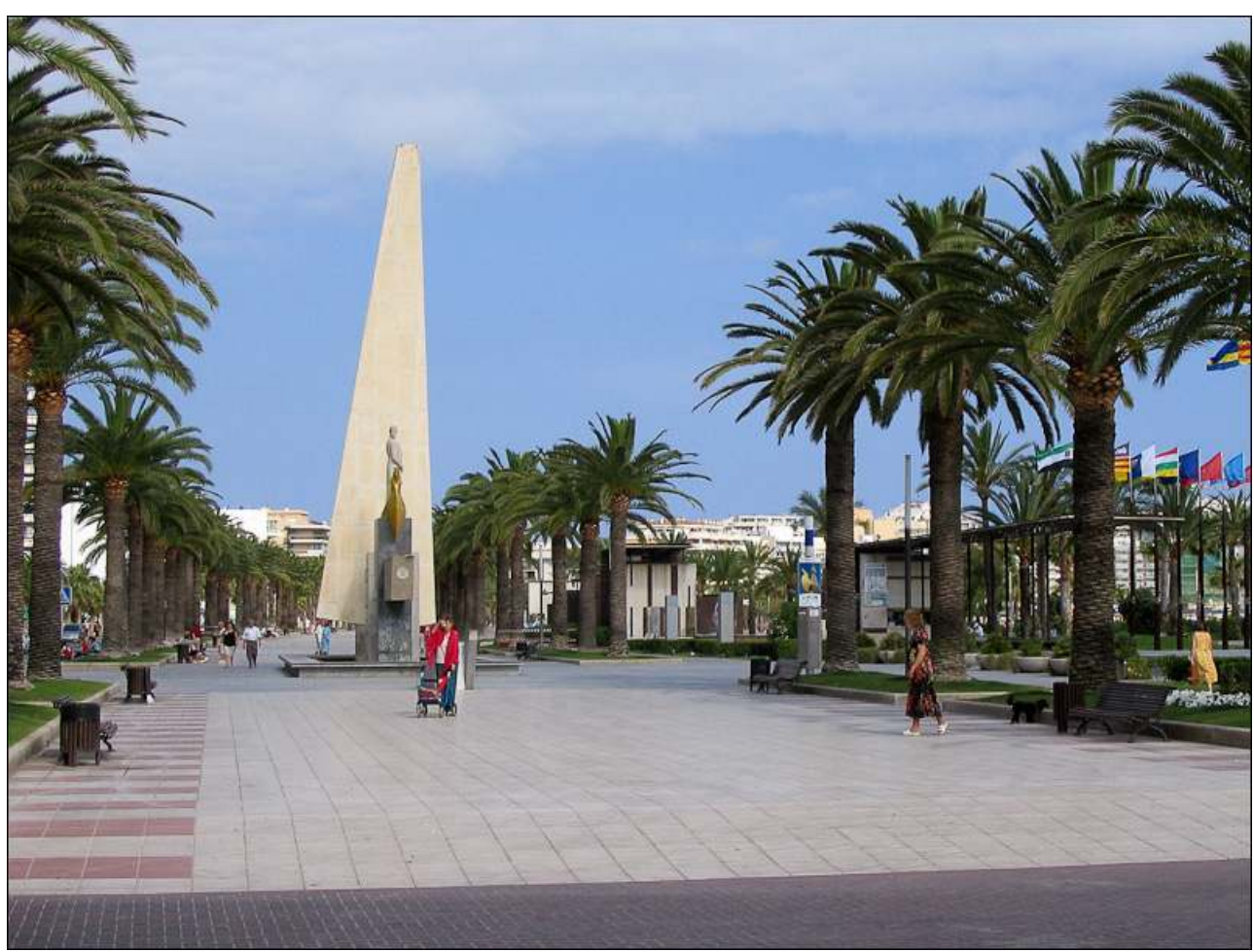

Auteur : Jean Rieucau, mai 2006. 
Illustration 10 - Faible boisement du parc-promenade, en raison de l'importance prise par les locaux d'entretien, les lampadaires, le mobilier urbain

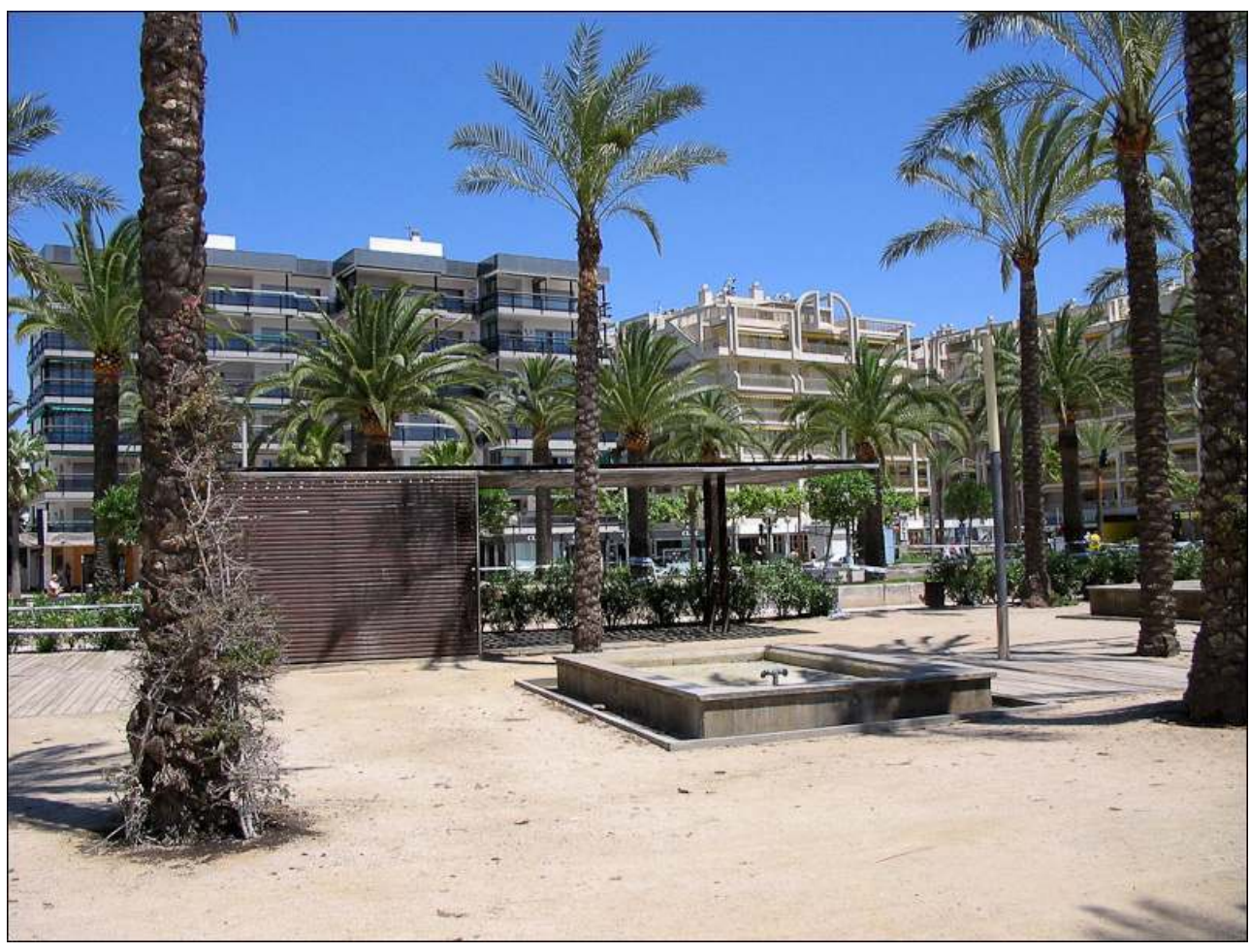

Auteur : Jean Rieucau, mai 2006.

En arrière du haut de plage, entre le parc et la plage, un labyrinthe ludique végétalisé (orangers, magnolias, araucarias, tamaris, lauriers blancs, bananiers, espaces gazonnés) a été aménagé pour fournir aux baigneurs au cours de la journée: ombre (palmiers, pergolas en bois bâchées, bancs publics), fraîcheur (sol en terre battue, parterre recouvert de lattes de bois, sol gazonné', bassins, pièces d'eau eau courante, eau sous pression des douches) et jeux (installations pour enfants, pétanque, aérobic, ping-pong), afin d'être un complément récréatif au balnéarisme stricto sensu. Au contact entre la promenade et le sable prend place dans la journée une concentration hétéroclite d'usagers de la plage (grands-parents, parents et enfants en bas âge dans leur poussette, couples de retraités, personnes âgées seules, handicapés, touristes à couleur de peau claire) qui recherche la proximité des jeux sécurisés pour enfants et les bosquets de palmiers (sol pour moitié gazonné, pour moitié recouvert de lattes de bois, munis de douches, de poubelles, pourvoyeurs de calme, d'ombre et de fraîcheur) (illustration 11). 


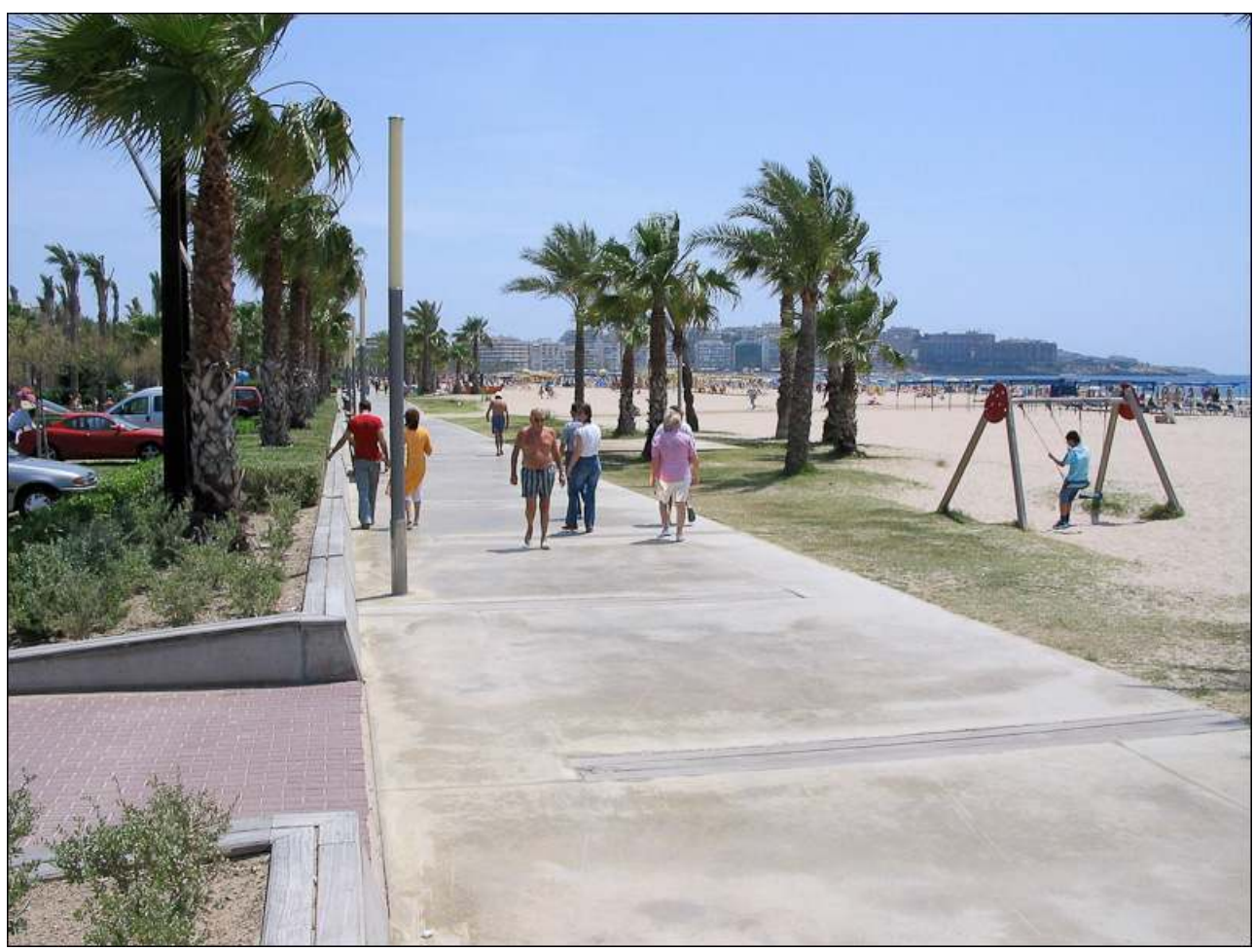

Auteur : Jean Rieucau, mai 2006.

La variété des usages récréatifs diurnes à l'intérieur du parc vise à enrichir, à étoffer (rencontrer, déjeuner, prendre le frais) le sol y playa fondé sur le bronzage et le bain de mer. La fréquentation diurne de la grande allée centrale du parc Jaume $\mathrm{I}^{\mathrm{er}}$ associe des touristes retraités, des grands-parents et leurs petits enfants, des familles. Il fonctionne tel un espace vert, public, de l'entre soi (fort clivage résidents permanents/touristes) peu générateur de confrontations de cultures et d'interrelations sociales.

objectifs des concepteurs du binôme parc/plage étaient multiples : limiter fortement la circulation automobile en bord de mer, renaturer la façade maritime, offrir un complément diurne et nocturne au sol y playa, mettre en place un usage nocturne de la plage, créer un interface entre la ville et la plage, déplacer l'urbanité déambulatoire nocturne, du centre-ville vers le front de mer. Le parc-promenade constitue une forme urbaine nouvelle, qui permet également d'enrichir la ressource touristique et de renouveler l'image touristico-récréative de la ville.

\section{Le paseo de la Segregació : promenade urbaine pour la ville « habitante »}

Dans le monde méditerranéen non musulman, la vie publique prend place dans la rue. L'ensemble de la société prend part à la promenade de début de soirée, en été, en Italie ( passegiatta), en Espagne (paseo). Le terme paseo, utilisé dans l'ensemble de l'Espagne ( rambla en Catalogne), constitue une dénomination générique et qualifie à la fois le lieu où l'on se promène, sur une distance variable de quelques dizaines de mètres à plusieurs kilomètres, mais également l'action de se promener : pasear (ramblear en Catalogne).Dans 
la péninsule ibérique, existe un goût pour la promenade publique (Berdoulay, Morales, op.cit.), fondé sur une forme d'urbanité déambulatoire, au sein d'espaces publics fortement fréquentés, dans lesquels la foule est indispensable (Berdoulay, Morales, op. cit. ) pour une mise en scène de la vie citadine. La promenade urbaine ibérique combine deux fonctions essentielles: permettre l'interaction sociale (sitio social), servir de lieu de rencontres (sitio de encuentro).

Le paseo de la Segregació (carte 1) constitue un des quatre paseos radiaux de la nouvelle ville située au nord (chaque nouveau quartier disposant d'un paseo). Cette promenade urbaine n'est pas en lien avec le tourisme, ni avec son image, à la différence du parcpromenade côtier. D'une longueur de 500 mètres, ce lien urbain s'insère dans une trame verte (peupliers, eucalyptus, conifères) qui contribue à aérer le nord de la ville et sert de liant piétonnier aux nouveaux services administratifs (écoles, mairie, médiathèque, théâtre, auditorium, nouvelle gare ferroviaire de la RENFE). Ce promenoirse compose d'une allée centrale (servant également de piste cyclable), sinueuse, ponctuée de bancs publics, bordée de deux voies de circulation automobile. Au delà des deux axes routiers, des massifs de peupliers, d'acacias, d'eucalyptus et de vieux oliviers, complètent cette promenade naturée. Le paseo dépourvu d'éléments construits à caractère culturel (sculptures) est régulièrement ponctué de vides, servant de lieu de rencontres. Sa végétation se compose d'acacias, de vieux oliviers ${ }^{10}$, de cyprès, de lauriers roses, d'espaces engazonnés. Cette promenade ne produit pas un paysage de nature dans la ville en raison d'une végétation trop diffuse, d'une nature trop domestiquée ${ }^{11}$, trop fonctionnelle bien qu'indigène, enfin en raison de la multiplicité des voies de circulation qui coupent dans sa longueur le déploiement du paseo. Si le végétal l'emporte sur le minéral, par contre les arbres sont de petite taille et non producteurs d'ombre.

\section{Le parc de la Ciutat : peu de mixité sociétale entre la ville touristique et la ville habitante}

Le parc de la Ciutat, aménagé en 1992 aux dépens de l'ancien terrain de camping de Salou, couvre $15750 \mathrm{~m}^{2}$. Cet espace vert, très enchâssé dans le tissu urbain, forme une oasis de nature jardinée dans la ville (Donadieu, op.cit.), un espace urbain de rafraîchissement (illustration 12). Aménagé sur trois niveaux, le parc comporte une palmeraie et un jardin botanique. Il est parcouru de canaux d'eau courante, encadré par des massifs de papyrus, bordé de magnolias, rythmé par des cascades. Il rassemble majoritairement des conifères de grande taille (pins et épicéas), privilégie le végétal (60\% de sa surface) et produit un paysage de parc urbain boisé (qualifié de parc forestier par la municipalité). Le boisement touffu domine, les concepteurs ayant exclu les éléments culturels (absence de statues, de sculptures, de kiosques à musique). Bien que situé à proximité d'une zone de villas et d'hôtels, peu éloigné du parc-promenade Jaume $1^{\mathrm{er}}$, il ne participe pas à la construction de l'image touristique de la station et demeure un espace public fortement naturé, non mis en tourisme (à l'exception de rares retraités de nationalité étrangère). Des populations catalanes ou espagnoles, du troisième âge, y recherchent la présence de l'eau, la fraîcheur, l'absence de mise en scène touristique garante du calme. L'absence des jeunes, des touristes, des populations étrangères, empêche toute forme de confrontations des cultures, de coprésence, d'interrelations, entre la ville touristique et la ville « habitante ». A la différence du parc-promenade Jaume $1^{\mathrm{er}}$, la foule n'est pas nécessaire, 
les visiteurs recherchent le silence (chants des oiseaux, bruit de l'eau courante), les pontons pour faire une pause, les bancs publics pour le repos.

Illustration 12 - Parc de la Ciutat

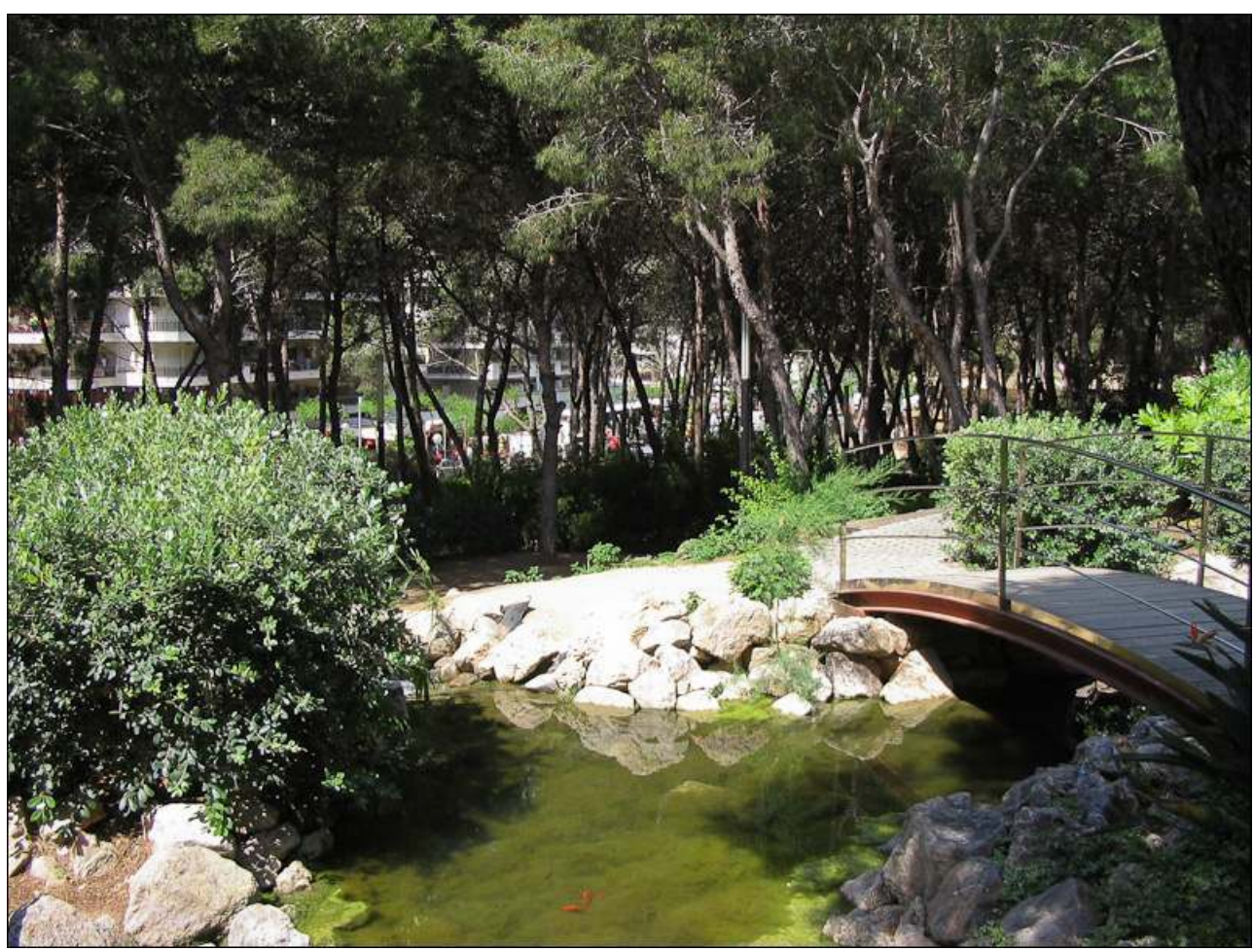

Espace vert enchâssé dans le tissu urbain, non mis en tourisme, ce qui garantit le silence, le repos des populations du troisième âge.

Auteur : Jean Rieucau, mai 2006.

\section{Conclusion}

Dans l'Espagne méditerranéenne, le tourisme de masse côtier a provoqué une urbanisation touristique anarchique, une altération des paysages et de l'environnement côtier. Pour adoucir la massification touristique, relancer des stations devenues obsolètes présentant une offre touristique indifférenciée, en partie délaissées par les clientèles de l'Europe du nord-ouest, les municipalités conduisent des opérations de récupération, de renouvellement urbain. Elles s'effectuent aux dépens d'espaces aménagés trop rapidement entre 1955 et 1960 (terrains de camping, hôtels, parcs de stationnement, établissements forains, pour certains aménagés sur les hauts de plage). Les espaces urbains ainsi récupérés font l'objet d'une renaturation (remplacement des hôtels établis sur la plage par des oasis de palmiers, substitution d'un parc-promenade à un parc de stationnement automobile, récupération d'espaces non bâtis sur les linéaires côtiers rocheux).

Dans ces villes-stations, urbanistes, paysagistes, sont confrontés aux rapports différenciés à l'espace public, des touristes et des résidents permanents, clivant la ville touristique et la ville «habitante ». Il en résulte une renaturation différenciée entre des lieux publics mis en tourisme, naturés au moyen d'une végétation décontextualisée et "mondialisée » (palmiers) et des espaces publics réservés aux résidents permanents (nouveaux paseos du 
nord de la ville) privilégiant une nature indigène, considérée comme " rassurante », faite d'espèces locales (oliviers, cyprès, acacias, eucalyptus, figuiers, lauriers roses). Ces espaces verts qui ne contribuent pas à la construction de l'image touristique excluent l'usage des palmiers et de végétaux exotiques, à la différence des promenades de front de mer, emblématiques du tourisme international.

\section{Illustration 13 - Front de mer de la station de la Pineda}

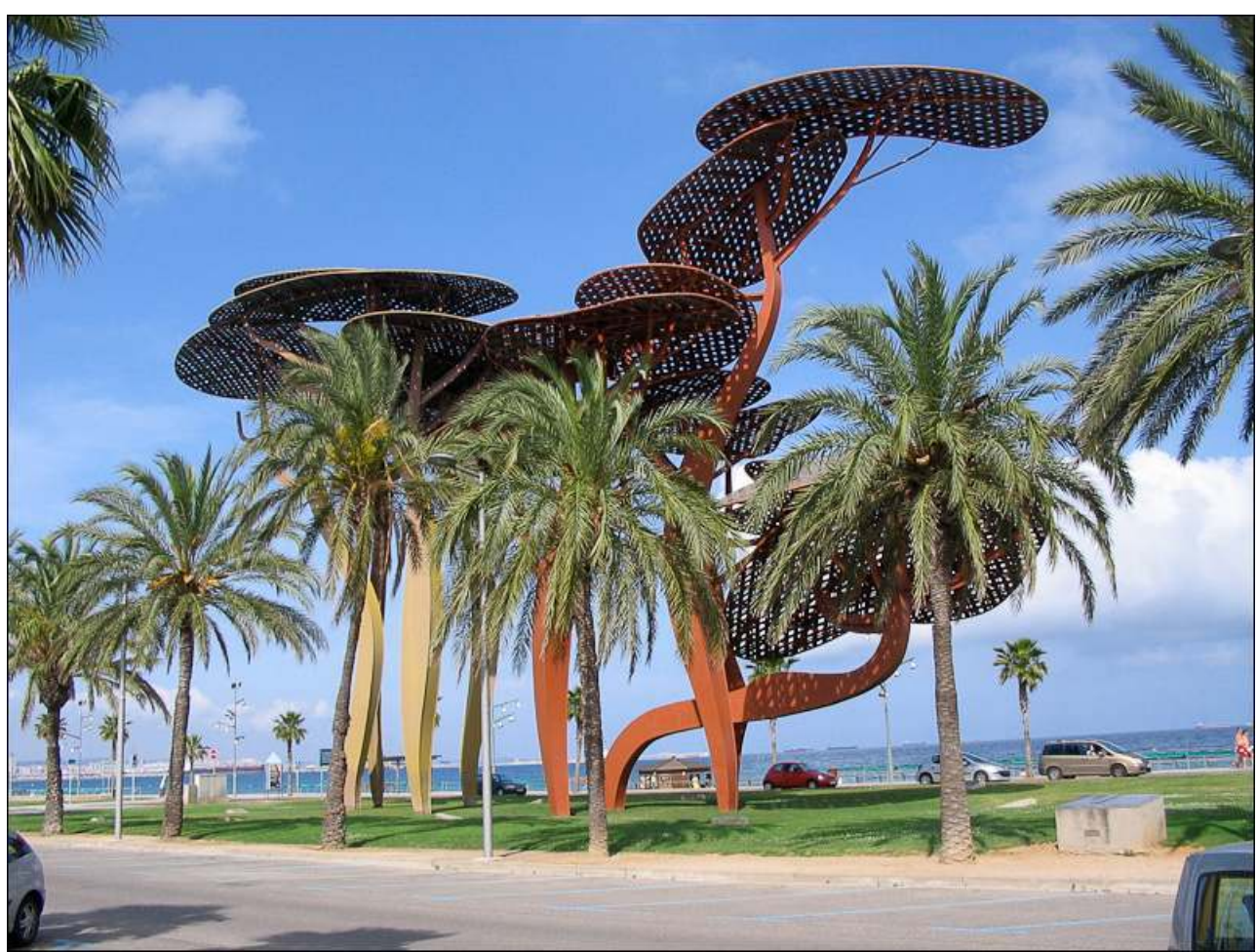

Implantation d'une nature factice au moyen de pins parasols métalliques géants.

Auteur : Jean Rieucau, juin 2010 
Illustration 14 - Une pinède factice dans un espace de récupération urbaine, aux dépens d'un ancien terrain de camping, sur le front de mer de la station de la Pineda

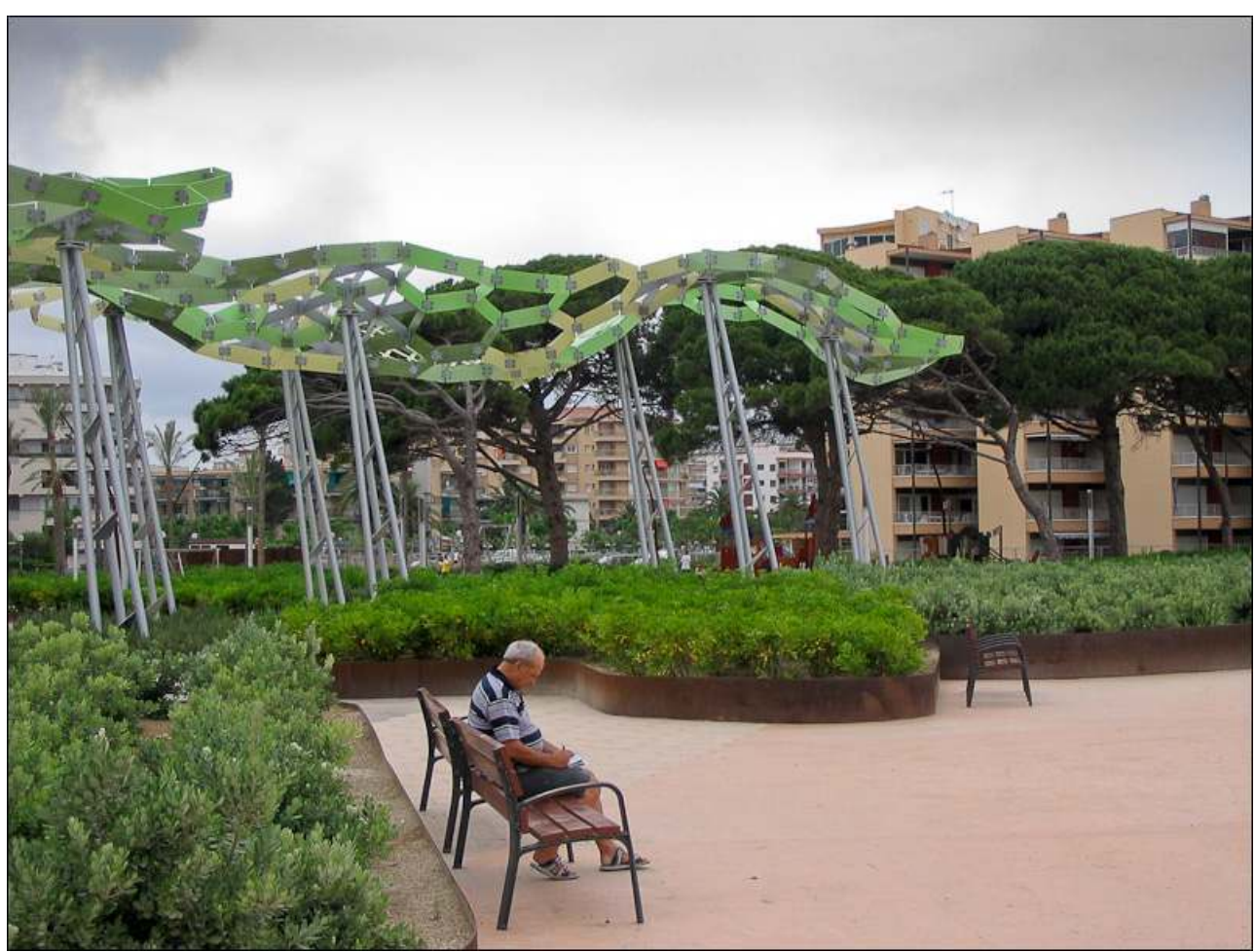

Auteur : Jean Rieucau, juin 2010 


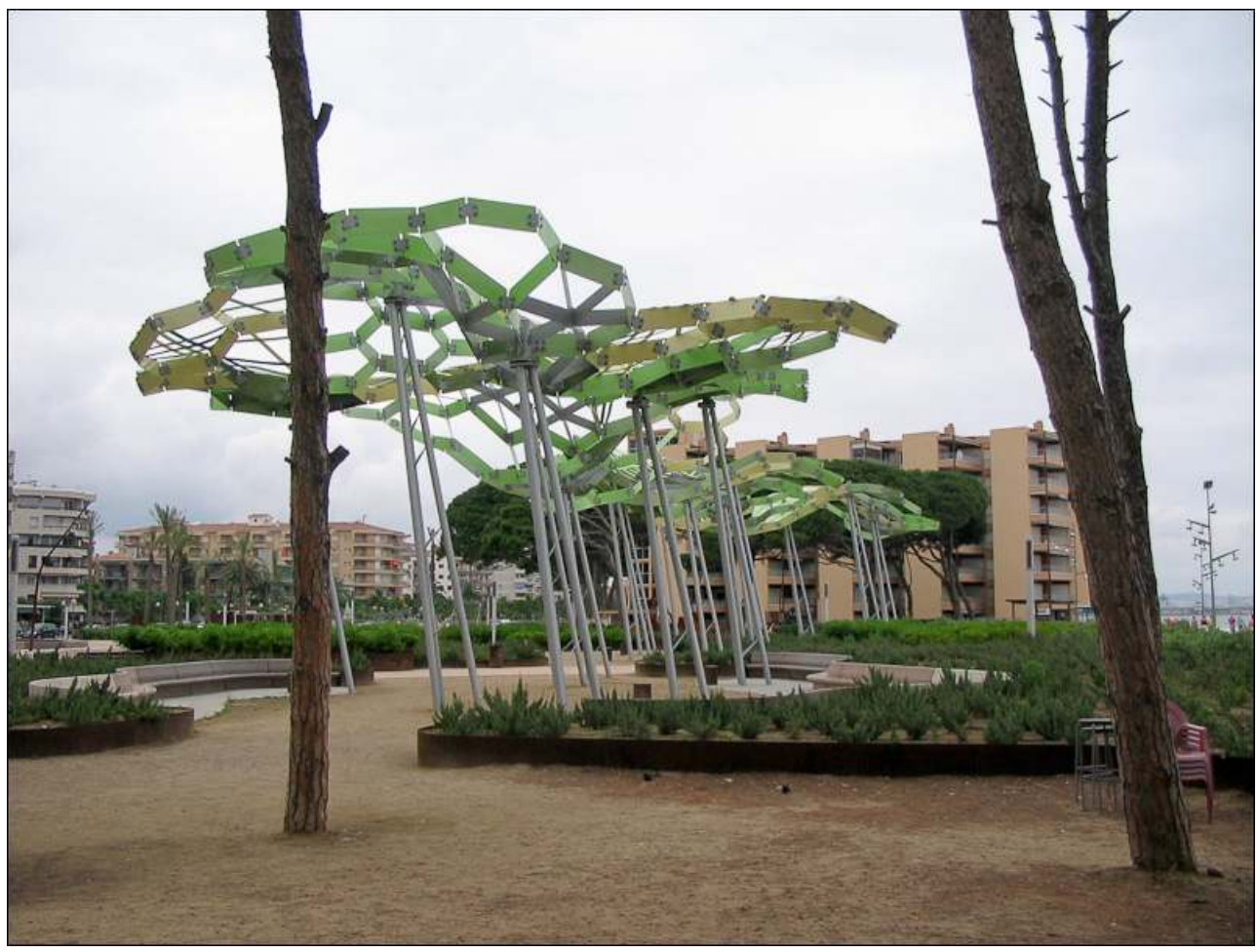

L'inclinaison des troncs de pins métalliques suggère l'intégration de la nature irréelle au sein de la nature réelle dans la station.

Auteur : Jean Rieucau, juin 2010.

\section{BIBLIOGRAPHIE}

Arnould P., 2006. Biodiversité : la confusion des chiffres et des territoires. Annales de Géographie, Paris, Armand Colin, ${ }^{\circ}$ 651, p. 528-549.

Berdoulay V., Morales, 1999. Espace public et culture : stratégies barcelonaises. Géographie et Cultures, l'Harmattan, n² 29, p. 79-94.

Bonnin Ph., 2010. Quand la nature s'urbanise, Natures urbanisées. Ethnologie Française, Paris, PUF, p. 581-587.

Claval, P., 1981. La logique des villes, Librairies techniques, Paris, 633 p.

Donadieu P., 2005. Entre urbanité et ruralité, la médiation paysagiste. Les Annales de la Recherche Urbaine, Paris, $n^{\circ} 85$, p. 7-15.

Coralli M., 2007. La plage de Cotonou, un lieu pour se montrer. Urbanisme, Paris, $\mathrm{n}^{\circ} 355$, p. 34-38.

Dorier Apprill E. (dir)., (2006). Ville et environnement. Paris, SEDES, 511 p. 
Estebanez J., 2006. Les jardins zoologiques et la ville : quelle nature pour le biodôme de Montréal ? Annales de Géographique, Paris, Armand Colin, n 652, p. 708-731.

Gillot G., 2006. Du paradis à Dream Park, les jardins dans le monde arabe : Damas, Le Caire, Rabat. Annales de Géographie, Armand Colin, Paris, nº65, p. 409-433.

Ghorra-Gobin C., 2001. Les espaces publics, capital social. Geocarrefour, Lyon, n 1, p. 5-11.

Lageiste J., Rieucau J. (dir.), 2008. La plage : un territoire atypique. Paris, l'Harmattan, Géographie et Cultures, $\mathrm{n}^{\circ}$ 67, $143 \mathrm{p}$.

Lévy J., Lussault M. (dir.), 2003. Dictionnaire de la Géographie et de l'espace des sociétés. Paris, Belin, $1034 \mathrm{p}$.

Merlin P., Choay F., 1996. Dictionnaire de l'urbanisme et de l'aménagement. Paris, Presses Universitaires de France, 863 p.

Miaux S., 2009. Corps urbains, mouvement et mise en scène. Géographie et Cultures, n 70, p. 3-6.

Péron F., Rieucau J. (dir.), 1996. La maritimité aujourd'hui. Paris,l'Harmattan, 336 p.

Rieucau J., 2000. La Grande-Motte, Ville permanente, ville saisonnière. Annales de Géographie, Armand Colin,Paris, $n^{\circ}$ 616, p. 631-654.

Rieucau J., 2006. La promenade maritime de la station aristocratique à la ville de l'âge postouristique. L'urbanité d'un espace public aux limites de l'oekoumène. L'Empreinte du tourisme, Contribution à l'identité du fait touristique, Rieucau J., Lageiste, J, (dir.), Paris, l'Harmattan, p. 121-175.

Rieucau J., Lageiste J., 2008. La plage un territoire singulier : entre hétérotopie et antimonde. In Lageiste J., Rieucau J. (dir.), La plage : un territoire atypique.Paris, l'Harmattan, Géographie et Cultures, $n^{\circ} 67$, p. 3-6.

Rieucau J., 2008. Vers des plages postbalnéaires au début du XXI ${ }^{\mathrm{e}}$ siècle. Entre domestication estivale et naturalité hivernale In Lageiste J., Rieucau J. (dir.), La plage : un territoire atypique.Paris, l'Harmattan, Géographie et Cultures, nº 67, p. 27-46.

Rieucau J., 2009. De la rue en sciences humaines, en architecture et dans l'urbanisme, au début du $\mathrm{XXI}^{\mathrm{e}}$ siècle. Lyon, Géocarrefour, $\mathrm{n}^{\circ}$ 3, p. 172-173.

Royoux D., Vassallo P., Zedda R., 2010. Vers une conciliation spatio-temporelle de la ville touristique et de la ville « habitante ». Paris, Cahiers Espaces, mars, p. 62-68.

Serpa A. (2004), Le parc urbain : un espace public dans la ville contemporaine ? Etude comparée Paris-Salvador de Bahia, Paris, l'Harmattan, Géographie et Cultures, n 52, p 91-104.

\section{NOTES}

1. Au sein des milieux hyperurbanisés, la plage maritime et l'avant-côte déploient une biodiversité saisonnièrement menacée. Ce milieu naturel, difficile à aménager, au contact terre/ mer, fait l'objet, en saison estivale, de tentatives de domestication, d'artificialisation, par les aménageurs, les urbanistes, les paysagistes. Par contre, en période hivernale, la plage reprend un caractère davantage naturel (recolonisation par les végétaux, par certains mollusques) (Rieucau, 2008).

2. L'Espagne méditerranéenne, depuis les années 1955, donne naissance à un modèle de tourisme, le sol y playa (soleil et plage) ou tourisme de plage, fondé sur la pratique du soleil, de la plage, de la mer. Ce tourisme, destiné à des populations de niveau social moyen, se caractérise 
par des investissements désordonnés, insuffisants en infrastructures, en équipements récréatifs, en aménagements touristiques. Il entraîne une prolifération touristique incontrôlée, un envahissement des littoraux, une altération des paysages et du milieu côtier.

3. En Europe, à partir de 1850 , des jardins privés s'ouvrent au public (jardins religieux, aristocratiques, royaux). Progressivement, l'ouverture de l'espace public triomphe face à l'enfermement des espaces privés (Donadieu, op. cit.), facilitant les interrelations et la mixité sociale. En Europe, le concept du jardin public confère une grande importance aux promenades et aux « espaces plantés ». En France, il naît sous le second Empire, avec le baron Haussmann. Antérieurement au XIXe siècle, les classes dirigeantes pratiquaient les jardins aristocratiques, principaux espaces verts des villes. Puis, ceux-ci se raréfient, à la suite de la disparition des jardins conventuels, de ceux des monastères, faisant apparaître la nécessité d'aménager de nouvelles coupures vertes. En Espagne, l'accession au pouvoir de la bourgeoisie urbaine contribue à accélérer la mise en place de promenades publiques (Rieucau, 2006).

4. Les figures de la nature en ville peuvent prendre des formes variées: arbres d'ornement, espaces forestiers, vignes, couloirs de faune, trames vertes, complétées par la gestion des friches, souvent siège d'une biodiversité méconnue (gestion confiée au paysagiste Gilles clément à Montpellier), toitures végétalisées.

5. Le port de plaisance, bien que de petite taille, a été aménagé au cœur de la station, fait rarissime sur le littoral méditerranéen espagnol, nécessitant le déplacement de l'activité halieutique dans la station voisine de Cambrils.

6. Les activités agricoles, aujourd'hui disparues de la ville occidentale, ont longtemps appartenu au quotidien urbain, en particulier dans la ville médiévale, qui conservait en son sein : espaces verts, jardins et animaux (Dorier Apprill, op. cit.). La ville maritime, investie ou non par le tourisme, conserve encore aujourd'hui, dans de nombreux cas, une fonction alimentaire, au travers du port de pêche.

7. Le parc constitue également un espace vert public, planté, mais de plus grande dimension que le jardin (Merlin, Choay, op. cit.). En Europe, le mouvement de création des parcs urbains s'appuie sur deux éléments : des raisons hygiéniques, ainsi qu'un mouvement de retour à la nature, propagé par le courant littéraire du romantisme (Merlin, Choay, op. cit.). En Amérique du nord, l'appellation parc naît dans les années 1860, aux Etats-Unis, et s'accompagne de l'émergence d'une sensibilité environnementale, que va propager le courant de réformisme social, qui contribue à diffuser une conception démocratique d'accès à la nature (Lévy, Lussault, op. cit.). Sur la façade orientale des Etats-Unis, se développe un mouvement de création de parcs urbains, sur le modèle de celui de Central Park de Manhattan, à New York (Lévy-Lussault, op. cit.).

8. Au début du XXI ${ }^{\mathrm{e}}$ siècle, la montée de l'insécurité dans les villes oriente la conception des espaces verts publics vers un moindre recours au végétal, favorisant les éléments minéraux (Serpa, 2004). Les parcs contemporains mettent en avant la monumentalité de leur conception, leur aspect fortement architecturé, ainsi qu'un nouveau rapport du binôme végétal/minéral (Serpa, op. cit.). Les nouveaux parcs comportent peu de végétation de grande taille et se composent de nombreux éléments construits. La nature urbaine y apparaît figée, théâtralisée (Serpa, op. cit.).

9. Les surfaces engazonnées font l'objet d'une remise en cause dans les villes, pour leur fort besoin en eau, pour leur pauvreté en valeur écologique. Dans les nouveaux parcs urbains, des prairies fleuries ou sèches (attirantes pour les hyménoptères: abeilles, guêpes, bourdons, frelons ; pour les lépidoptères : papillons, pour l'avifaune), remplacent les pelouses irriguées.

10. En 1989, pour commémorer la création de Salou, seize oliviers «monumentaux » ont été plantés le long du paseo de la Segregació.

11. Certains paysagistes peuvent faire des choix plus radicaux en matière d'implantation d'une nature irréelle. Sur le front de mer de la station voisine de Salou : la Pineda (toponyme signifiant la pinède), la municipalité a eu recours à une nature allogène, factice, au moyen de pins parasols 
métalliques géants (cf photo 13), pour scénographier la promenade maritime. Dans la même station, sur un haut de plage dévégétalisé, les paysagistes pouvaient replanter des pins, mais, ils ont choisi l'implantation d'une nature artificielle, métallique, représentant une pinède, allant jusqu'à l'utilisation de la forte inclinaison, des troncs des pins en métal (cf illustrations 14 et 15).

\section{RÉSUMÉS}

Les espaces publics naturés deviennent un enjeu de l'attractivité touristique des stations côtières sur le littoral méditerranéen espagnol. Les municipalités conduisent des politiques de récupération urbaine sur les plages, les hauts de plage, le long du linéaire côtier, aux dépens d'une urbanisation anarchique, héritée des années 1960 (hôtels, terrains de camping). Les paysagistes confrontés aux clivages entre la ville touristique et la ville « habitante » utilisent une végétation allogène, décontextualisée (palmiers), pour les espaces publics mis en tourisme et recourent à une nature indigène (oliviers, cyprès) pour les espaces communs des résidents permanents.

The forested public areas become a stake of the touristic appeal of coastal destinations on the Mediterranean coast of Spain. The municipalities steer the urban recovery policies on the beaches, beach heights, along the coastal line, at the expense of anarchical urbanization, inherited from the years 1960 (hotels, camping ground). The landscapers, confronted with the split between touristic city and "resident" city, use exotic vegetation, out of context (palm tree) for public areas developed into tourism, and resort to indigenous nature (olive trees, cypress) for common areas for the permanent residents.

\section{INDEX}

Keywords : Mediterranean coast, reforestation, reforested public areas, Spain, tourism, urban recovery, urbanity

Mots-clés : côte méditerranéenne, espaces publics naturés, Espagne, récupération urbaine, renaturation, tourisme, urbanité

\section{AUTEUR}

\section{JEAN RIEUCAU}

Jean Rieucau est professeur des Universités, membre de l'UMR 5600 Environnement, ville, société (CNRS)/Université Lumière Lyon 2. jean.rieucau@wanadoo.fr 